\title{
Stability Analysis of Switched Systems with Time-Varying Discontinuous Delays
}

Frédéric Mazenc

\author{
Michael Malisoff
}

\author{
Hitay Özbay
}

\begin{abstract}
A new technique is proposed to ensure global asymptotic stability for nonlinear switched time-varying systems with time-varying discontinuous delays. It uses an adaptation of Halanay's inequality to switched systems and a recent trajectory based technique. The result is applied to a family of linear time-varying systems with time-varying delays.
\end{abstract}

Index Terms-Switched systems, nonlinear, delays

\section{INTRODUCTION}

Switched systems in continuous-time are systems that include discrete switching events and a switching signal that specify which one of its subsystems is utilized at each instant [12]. They are important for many applications, including communication networks; see [2], [10], and [14]. Delays are also frequently present in engineering processes, either in outputs (measurement delays) or control inputs (actuator delays) in feedback loops. In certain applications, the time delays can be time-varying and discontinuous, e.g., in control over a network, where failures in links or congestion can lead to sudden changes in the routing, which can cause the returntrip-time to change abruptly. Here and in what follows, we use discontinuous to mean not necessarily continuous.

Analyzing the stability of systems with discontinuous delays can be difficult, even for systems without switching. To cope with discontinuous delays, one approach involves representing the systems as switched systems and then using switched systems theory. This motivated [12] and [15], which involve switched nonlinear systems with lumped delays, [1] (which provided improved dwell time values under constant delays and delay bounds), and [11] (which has sufficient conditions for stability with lower bounds on the average dwell time and upper bounds on the delays). See [13] for non-delayed switched systems, under an assumption on a stable convex combination of their subsystems.

Here, we study switched systems, using a very different approach. Our main new result (in Section II) for nonlinear systems combines Halanay's inequality [3] with [8]. A key advantage of our work is that it applies to broad classes of systems including time-varying systems with discontinuous

Mazenc and Özbay were supported by a PHC Bosphore 2016 project with reference numbers 35634QM (France) and EEEAG-115E820 (TÜBİTAK Turkey). Malisoff was supported by NSF Grant 1408295.

Mazenc is with EPI DISCO INRIA-Saclay, Laboratoire des Signaux et Systèmes (L2S, UMR CNRS 8506), CNRS, CentraleSupélec, Université Paris-Sud, 3 rue Joliot Curie, 91192, Gif-sur-Yvette, France, frederic.mazenc@12s.centralesupelec.fr.

Malisoff is with the Department of Mathematics, Louisiana State University, Baton Rouge, LA 70803-4918, USA, malisoff@1su.edu.

Özbay is with the Department of Electrical and Electronics Engineering, Bilkent University, Ankara 06800, Turkey, hitay@bilkent.edu.tr. delays and switching. This contrasts with the literature, since many works assume that the time-varying delays are continuously differentiable. Our results also differ from the valuable work [4], which has conditions on the activation time of the unstable systems and on the average dwell time, which we do not require here. Our key assumption is on the switching signal. This limits the number of switchings on certain intervals, but a key novel feature is that we do not impose an upper bound on the supremum of the delay.

As in [4], we do not assume that all of the subsystems of the switched system are stable, nor we require a common Lyapunov function for the subsystems. This is valuable, because there are techniques available for systems with common Lyapunov functions, but, in many cases, common Lyapunov functions do not exist, and the presence of a time-varying delay precludes the use of standard invariance principles. Usually, the existence of a common Lyapunov function implies stability for any switching signal, and we aim to establish stability results under restricted switching signals because only this type of result makes it possible to solve our problems for time-varying systems that have timevarying delays; see Remark 1 below.

We will use the following notation. We omit arguments of functions when the arguments are clear from the context, and the dimensions of our Euclidean spaces are arbitrary unless otherwise noted. Set $\mathbb{N}=\{1,2, \ldots\}$ and $\mathbb{Z}_{>0}=\mathbb{N} \cup\{0\}$. We use $|\cdot|$ to denote the usual Euclidean norm, and $|\phi|_{J}$ to denote the supremum of any function $\phi$ over any interval $J$ in its domain, and $I_{n}$ is the identity matrix in any dimension $n$. We let $f\left(t^{-}\right)$denote the limit from the left of functions $f$ at points $t$ where the left limit exists. For any constant $\tau_{b}>0$, let $C_{\text {in }}$ be the set of all continuous functions $\phi$ : $\left[-\tau_{b}, 0\right] \rightarrow \mathbb{R}^{n}$, which we call the set of all initial functions. We define $\Xi_{t} \in C_{\text {in }}$ by $\Xi_{t}(s)=\Xi(t+s)$ for all $\Xi, s \leq 0$, and $t \geq 0$ for which the equality is defined. Let $\mathcal{K}_{\infty}$ be the set of all continuous functions $g:[0,+\infty) \rightarrow[0,+\infty)$ such that $g(0)=0, g$ is strictly increasing, and $\lim _{s \rightarrow+\infty} g(s)=+\infty$.

\section{Fundamental Result}

\section{A. Systems and Assumptions}

Consider any nonlinear time-varying switched system with an unknown piecewise continuous delay $\tau:[0,+\infty) \rightarrow$ $\left[0, \tau_{b}\right]$, represented by

$$
\dot{x}(t)=f_{\sigma(t)}(t, x(t-\tau(t)))
$$

where $x$ is valued in $\mathbb{R}^{n}, \sigma:[0,+\infty) \rightarrow\{1, \ldots, k\}$ is called the switching signal, $k \in \mathbb{N}$ is arbitrary, each $f_{i}$ is locally 
Lipschitz with respect to its second argument and piecewise continuous with respect to its first argument $t$ for all $i \in$ $\{1, \ldots, k\}, f_{i}(t, 0)=0$ for all $t \geq 0$ and $i, \tau_{b}>0$ is a known constant, and the initial functions $\phi$ are in $C_{\mathrm{in}}$.

Choose $\left\{t_{i}\right\}$ to be the switching sequence of nonnegative real numbers associated with $\sigma$, i.e., the times $\sigma$ changes to a new value, with $t_{0}=0$ and $\sigma(t)=\sigma\left(t_{i}\right)$ for all $t \in\left[t_{i}, t_{i+1}\right)$. Assume that there are two constants $\mathcal{T}_{1}$ and $\mathcal{T}_{2}$ such that

$$
0<\mathcal{T}_{1}<t_{i+1}-t_{i} \leq \mathcal{T}_{2} \text { for all } i \in \mathbb{Z}_{\geq 0} .
$$

The constant $\mathcal{T}_{1}$ is usually called the (minimal) dwell time. See [9], and [5] for design methods for minimizing dwell times and ensuring stability of delay systems. These papers develop stability results in the form "if $\mathcal{T}_{1}<t_{i+1}-t_{i}$ for all $i$, then the system is stable" under certain technical assumptions (usually requiring all subsystems to be stable) [1]. Assume:

Assumption 1: There are $k$ absolutely continuous functions $V_{j}:[0,+\infty) \times C_{\mathrm{in}} \rightarrow[0,+\infty)$ for $j=1,2, \ldots, k$, real numbers $\alpha_{1}, \ldots, \alpha_{k}$, nonnegative constants $\beta_{1}, \ldots, \beta_{k}$, a continuous function $W: \mathbb{R}^{n} \rightarrow[0,+\infty)$, and class $\mathcal{K}_{\infty}$ functions $\chi_{1}$ and $\chi_{2}$ such that

$$
\chi_{1}(|\phi(0)|) \leq V_{j}(t, \phi) \leq \chi_{2}\left(|\phi|_{\infty}\right)
$$

hold for all $\phi \in C_{\mathrm{in}}, t \in[0,+\infty)$, and $j \in\{1,2, \ldots, k\}$ and such that for all $i \in \mathbb{Z}_{\geq 0}$, the condition

$$
\dot{V}_{\sigma\left(t_{i}\right)}(t) \leq \alpha_{\sigma\left(t_{i}\right)} V_{\sigma\left(t_{i}\right)}\left(t, x_{t}\right)+\beta_{\sigma\left(t_{i}\right)} \sup _{\ell \in\left[t-\tau_{b}, t\right]} W(x(\ell))
$$

holds along all trajectories of

$$
\dot{x}(t)=f_{\sigma\left(t_{i}\right)}(t, x(t-\tau(t)))
$$

for almost all $t \in\left[t_{i}, t_{i+1}\right)$.

Assumption 2: The functions $V_{j}$ and $W$ from Assumption 1 admit a constant $\mu>1$ such that for all $t \geq 0$, we have

$$
W(\phi(0)) \leq V_{1}(t, \phi) \text { and } V_{i}(t, \phi) \leq \mu V_{j}(t, \phi)
$$

for all $\phi \in C_{\text {in }}$ and all $i$ and $j$ in $\{1, \ldots, k\}$.

Assumption 3: There are constants

$$
T \geq \tau_{b}+\mathcal{T}_{2}
$$

and $\lambda(T)>0$ such that the inequality

$$
\int_{t-T}^{t} \alpha_{\sigma(\ell)} \mathrm{d} \ell \leq-\lambda(T)
$$

holds for all $t \geq T$.

With the above notation, we fix a constant $\nu \geq 0$ such that

$$
\int_{t-T}^{t} \beta_{\sigma(s)} e^{\int_{s}^{t} \alpha_{\sigma(s)} \mathrm{d} \ell} \mathrm{d} s \leq \nu \text { for all } t \geq T .
$$

For instance, we can take $\nu=T\left(\max _{i} \beta_{i}\right) e^{T \max _{i}\left|\alpha_{i}\right|}$. Let $N(r, t)$ be the number of switching instants $t_{i}$ on $[t-r, t)$ for all $r \in(0, t]$ and $t>0$, and $L(r)=\sup _{t \geq 0} N(r, t)$ (which is finite, by (2)). Our stability condition is:

Assumption 4: The inequality

$$
\mu^{L(T)+1} e^{-\lambda(T)}+\left[\frac{\mu^{L(T)+2}-1}{\mu-1}-L(T)-1\right] \nu \mu<1
$$

is satisfied, with the notation from Assumptions 1-3.

Remark 1: We allow some systems $\dot{x}(t)=f_{i}(t, x(t-$ $\tau(t))$ ) to be unstable, since the $\alpha_{i}$ 's can be positive or negative. The instability of the unstable subsystems should be compensated by the stability of other subsystems, so we cannot extend our result to switchings without restriction. Our work contrasts with the work [4] on cases where our $\beta_{i}$ 's are independent of the switching instants.

Remark 2: When one knows a common Lyapunov functional, i.e., $V_{1}=V_{j}$ for all $j \in\{2, \ldots, k\}$, the problems solved below can be solved by earlier methods [16]. Recall that globally uniformly exponential stability of a switched system does not imply that its subsystems have a common Lyapunov function; see [6, Section 2.1.5]. Also, if a switched system is input-to-state stable (or ISS) under arbitrary switching, then uniform (with respect to the switching signals) ISS is equivalent to the existence of a common ISS Lyapunov function; see [7]. Requiring a constant $\mu>1$ that satisfies the conditions from (5) in Assumption 2 is standard [6].

\section{B. Statement and Sketch of Proof of Main Result}

Theorem 1: If the switched system (1) satisfies Assumptions 1-4, then the origin of (1) is a globally uniformly asymptotically stable equilibrium.

Proof. (Sketch.) We set $a(t)=\alpha_{\sigma(t)}, b(t)=\beta_{\sigma(t)}$, and $U(t, \phi)=V_{\sigma(t)}(t, \phi)$. By induction, one can prove that (1) is forward complete. This follows by combining the decay estimates on the $V_{i}$ 's from Assumption 1 and using (5) on each interval $\left[t_{i}, t_{i+1}\right)$, and applying Gronwall's inequality to $\mathcal{F}(t)=\sup _{\ell \in[0, t]} V_{\sigma\left(t_{0}\right)}(\ell, x(\ell))$. Assumption 1 implies that for all $\ell \in \mathbb{Z}_{\geq 0}, r \in\left[t_{\ell}, t_{\ell+1}\right)$, and $t_{*} \in\left[t_{\ell}, r\right]$, we have

$$
\begin{aligned}
& U\left(r, x_{r}\right) \leq e^{\int_{t_{*}}^{r} a(s) \mathrm{d} s} U\left(t_{*}, x_{t_{*}}\right) \\
& +\int_{t_{*}}^{r} b(s) e^{\int_{s}^{r} a(r) \mathrm{d} r} \sup _{p \in\left[s-\tau_{b}, s\right]} W(x(p)) \mathrm{d} s .
\end{aligned}
$$

Next, let $t \geq T+\tau_{b}, q \geq 0$, and $j \in \mathbb{Z}_{\geq 0}$ be such that $t \in\left[t_{j}, t_{j+1}\right), j-1 \geq q$ and $t-T \in\left[t_{j-q-1}, t_{j-q}\right)$. We first consider the case where $q \geq 2$; later in the proof, we explain the changes needed to cover cases where $q \in\{0,1\}$. We use the simplifying notation $w(t)=\sup _{p \in\left[t-\tau_{b}, t\right]} W(x(p))$,

$$
\mathcal{E}(\ell, t)=e^{\int_{\ell}^{t} a(s) \mathrm{d} s}, \text { and } \mathcal{G}_{i}=\mu \mathcal{E}\left(t_{i-1}, t_{i}\right) .
$$

Using (10) with $\ell=j, t_{*}=t_{j}$ and $r=t$, and $U(t)$ to mean $U\left(t, x_{t}\right)$, we use (5) to get

$$
\begin{aligned}
U(t) & \leq \mathcal{E}\left(t_{j}, t\right) U\left(t_{j}\right)+b(t) \int_{t_{j}}^{t} \mathcal{E}(\ell, t) w(\ell) \mathrm{d} \ell \\
& \leq \mu \mathcal{E}\left(t_{j}, t\right) U\left(t_{j}^{-}\right)+\int_{t_{j}}^{t} b(\ell) \mathcal{E}(\ell, t) w(\ell) \mathrm{d} \ell .
\end{aligned}
$$

By similar reasoning, taking $\ell=j-1$ in (10) (and the limit $r \rightarrow t_{j}^{-}$from the left in (10)), and then using (5) from Assumption 2 and the nonnegativity of the $\mathcal{G}_{j}$ 's, we obtain

$$
U\left(t_{j}^{-}\right) \leq \mathcal{G}_{j} U\left(t_{j-1}^{-}\right)+\int_{t_{j-1}}^{t_{j}} b(\ell) \mathcal{E}\left(\ell, t_{j}\right) w(\ell) \mathrm{d} \ell
$$

and by similar reasoning, for any $p \in\{2, \ldots, q\}$, we have

$$
\begin{aligned}
& \prod_{r=j-p+2}^{j} \mathcal{G}_{r} U\left(t_{j-p+1}^{-}\right) \leq \prod_{r=j-p+1}^{j} \mathcal{G}_{r} U\left(t_{j-p}^{-}\right) \\
+ & \prod_{r=j-p+2}^{j} \mathcal{G}_{r} \int_{t_{j-p}}^{t_{j-p+1}} b(\ell) \mathcal{E}\left(\ell, t_{j-p+1}\right) w(\ell) \mathrm{d} \ell .
\end{aligned}
$$


By moving the left side term in (14) to the right side of (14), and then summing the inequalities in (13)-(14), we obtain

$$
\begin{aligned}
& U\left(t_{j}^{-}\right) \leq \prod_{r=j-q+1}^{j} \mathcal{G}_{r} U\left(t_{j-q}^{-}\right) \\
& +\int_{t_{j-1}}^{t_{j}} b(\ell) \mathcal{E}\left(\ell, t_{j}\right) w(\ell) \mathrm{d} \ell+\mathcal{G}_{j} \int_{t_{j-2}}^{t_{j-1}} b(\ell) \mathcal{E}\left(\ell, t_{j-1}\right) w(\ell) \mathrm{d} \ell \\
& +\ldots+\prod_{r=j-q+2}^{j} \mathcal{G}_{r} \int_{t_{j-q}}^{t_{j-q+1}} b(\ell) \mathcal{E}\left(\ell, t_{j-q+1}\right) w(\ell) \mathrm{d} \ell .
\end{aligned}
$$

Also, using (10) with the choices $\ell=j-q-1$ and $t_{*}=t-T$ (and by letting $r \rightarrow t_{j-q}^{-}$in (10)), we obtain

$$
\begin{aligned}
U\left(t_{j-q}^{-}\right) \leq & \mathcal{E}\left(t-T, t_{j-q}\right) U(t-T) \\
& +\int_{t-T}^{t_{j-q}} b(\ell) \mathcal{E}\left(\ell, t_{j-q}\right) w(\ell) \mathrm{d} \ell,
\end{aligned}
$$

since $t-T \in\left[t_{j-q-1}, t_{j-q}\right)$.

Hence, if $q \geq 2$, then our formulas for the $\mathcal{G}_{i}$ 's from (11) give

$$
\begin{aligned}
& U\left(t_{j}^{-}\right) \leq \mu^{q} \mathcal{E}\left(t_{j-q}, t_{j}\right) U\left(t_{j-q}^{-}\right) \\
& +\int_{t_{j-1}}^{t_{j}} b(\ell) \mathcal{E}\left(\ell, t_{j}\right) w(\ell) \mathrm{d} \ell \\
& +\left\{\mu \int_{t_{j-2}}^{t_{j-1}} b(\ell) \mathcal{E}\left(\ell, t_{j}\right) w(\ell) \mathrm{d} \ell\right. \\
& \left.+\ldots+\mu^{q-1} \int_{t_{j-q}}^{t_{j-q+1}} b(\ell) \mathcal{E}\left(\ell, t_{j}\right) w(\ell) \mathrm{d} \ell\right\} .
\end{aligned}
$$

Using (15) to upper bound the $U\left(t_{j-q}^{-}\right)$in (16), we conclude that if $q \geq 2$, then

$$
\begin{aligned}
& U\left(t_{j}^{-}\right) \leq \mu^{q} \mathcal{E}\left(t-T, t_{j}\right) U(t-T) \\
& +\int_{t_{j-1}}^{t_{j}} b(\ell) \mathcal{E}\left(\ell, t_{j}\right) w(\ell) \mathrm{d} \ell+\left\{\mu \int_{t_{j-2}}^{t_{j-1}} b(\ell) \mathcal{E}\left(\ell, t_{j}\right) w(\ell) \mathrm{d} \ell\right. \\
& \left.+\ldots+\mu^{q-1} \int_{t_{j-q}}^{t_{j-q}} b(\ell) \mathcal{E}\left(\ell, t_{j}\right) w(\ell) \mathrm{d} \ell\right\} \\
& +\mu^{q} \int_{t_{-T}}^{t_{j-q}} b(\ell) \mathcal{E}\left(\ell, t_{j}\right) w(\ell) \mathrm{d} \ell,
\end{aligned}
$$

which we can use to upper bound $U\left(t_{j}^{-}\right)$in (12) to get

$$
\begin{aligned}
& U(t) \leq \mu^{q+1} e^{-\lambda(T)} U(t-T) \\
& +\Lambda(t) \sup _{\ell \in\left[t-\tau_{b}-T, t\right]} W(x(\ell)),
\end{aligned}
$$

where

$$
\begin{aligned}
& \Lambda(t)=\int_{t_{j}}^{t} b(\ell) \mathcal{E}(\ell, t) \mathrm{d} \ell+\mu \int_{t_{j-1}}^{t_{j}} b(\ell) \mathcal{E}(\ell, t) \mathrm{d} \ell \\
& +\left\{\mu^{2} \int_{t_{j-2}}^{t_{j-1}} b(\ell) \mathcal{E}(\ell, t) \mathrm{d} \ell+\ldots+\mu^{q} \int_{t_{j-q}}^{t_{j-q+1}} b(\ell) \mathcal{E}(\ell, t) \mathrm{d} \ell\right\} \\
& +\mu^{q+1} \int_{t-T}^{t_{j-q}} b(\ell) \mathcal{E}(\ell, t) \mathrm{d} \ell,
\end{aligned}
$$

by (7). Since $\mu>1$ and $q \leq L(T)$, our condition (8) on $\nu$ implies that if $q \geq 2$, then

$$
\begin{aligned}
\Lambda(t) \leq & \int_{t-T}^{t} b(\ell) e^{\int_{\ell}^{t} a(s) \mathrm{d} s} \mathrm{~d} \ell+\left[\mu-1+\mu^{2}-1\right. \\
& \left.+\ldots+\mu^{L(T)+1}-1\right] \int_{t-T}^{t} b(\ell) e^{\int_{\ell}^{t} a(s) \mathrm{d} s} \mathrm{~d} \ell \\
\leq & {\left[\frac{\mu^{L(T)+2}-1}{\mu-1}-L(T)-1\right] \nu, }
\end{aligned}
$$

by the formula for the geometric sum, which we can combine with (17) to obtain

$$
\begin{aligned}
& U\left(t, x_{t}\right) \leq \mu^{L(T)+1} e^{-\lambda(T)} U\left(t-T, x_{t-T}\right) \\
& +\left[\frac{\mu^{L(T)+2}-1}{\mu-1}-L(T)-1\right] \nu \mu \sup _{\ell \in\left[t-\tau_{b}-T, t\right]} U\left(\ell, x_{\ell}\right)
\end{aligned}
$$

for all $t \geq T+\tau_{b}$.

On the other hand, if $q=0$, then we can use (15) to upper bound $U\left(t_{j}^{-}\right)$in (12) to obtain (19) in this case as well. Finally, if $q=1$, then (19) again follows, by arguing as above except with the quantities in curly braces from (16) through (17) removed. We can therefore use Assumption 4 and [8, Lemma 1] (with

$$
T^{*}=T+\tau_{b} \text { and } w(\ell)=U\left(\ell+T+\tau_{b}\right)
$$

in the lemma) to obtain an exponential decay estimate on $U\left(t, x_{t}\right)$ that is valid for all $t \geq T+\tau_{b}$. Also, we can use Gronwall's inequality to obtain an exponential decay estimate on $U\left(t, x_{t}\right)$ that is valid for all $t \in\left[0, T+\tau_{b}\right]$ (by applying Gronwall's inequality on successive intervals of the form $\left[t_{j}, t_{j+1}\right)$, and then multiplying the resulting right side by $\left.e^{T+\tau_{b}-t}\right)$. The global asymptotic stability estimate now follows from (3).

\section{Application to Linear Time Varying Systems}

We apply our main result to a fundamental family of linear time-varying (or LTV) systems with time-varying lumped delays. For simplicity, we do not assume that these systems have switches, but we illustrate connections with switched systems. We continue the notation of Section II.

\section{A. Problem Definition and Preliminary Remarks}

Consider the system

$$
\dot{x}(t)=A(t) x(t)+B(t) x(t-\tau(t)) .
$$

We first make this assumption (but see Remark 3 for generalizations, where the periodicity condition is removed):

Assumption 5: The functions $A, B$ and $\tau$ are piecewise $C^{1}$ and bounded and all three have the same period $\mathcal{P}>0$.

We can therefore fix constants $a_{b}, b_{b}$, and $\tau_{b}$ such that

$$
|A(t)| \leq a_{b},|B(t)| \leq b_{b}, \text { and } \tau(t) \leq \tau_{b}
$$

hold for all $t \geq 0$. We can also fix a constant $k \in \mathbb{N}$ and nonnegative constants $\bar{a}, \bar{b}$, an $\bar{\tau}$ such that the triples

$$
\left(A_{i}, B_{i}, \tau_{i}\right)=(A(i \mathcal{P} / k), B(i \mathcal{P} / k), \tau(i \mathcal{P} / k))
$$

for $i=1,2, \ldots, k$ satisfy

$$
\begin{aligned}
& \left|A(t)-A_{\sigma(t)}\right| \leq \bar{a},\left|B(t)-B_{\sigma(t)}\right| \leq \bar{b}, \\
& \text { and }\left|\tau(t)-\tau_{\sigma(t)}\right| \leq \bar{\tau} \text { for all } t \geq 0,
\end{aligned}
$$

where the switching signal $\sigma$ is defined by $\sigma(t)=i$ for all $t \in[i \mathcal{P} / k,(i+1) \mathcal{P} / k)$ and $i \in\{0, \ldots, k-1\}$ and has period $\mathcal{P}$, so (2) holds for some constants $\mathcal{T}_{i}$. For the preceding choices of $k, \sigma, \bar{a}, \bar{b}, \bar{\tau}, a_{b}, b_{b}, \mathcal{T}_{2}, \tau_{b}$, and the triples $\left(A_{i}, B_{i}, \tau_{i}\right)$, our next two assumptions are: 
Assumption 6: For each $i \in\{1, \ldots, k\}$, there exist an absolutely continuous function $V_{i}: C_{\mathrm{in}} \rightarrow[0,+\infty)$, a real constant $\alpha_{i}$, and a positive constant $\gamma_{i}$ such that the time derivative of $V_{i}$ along all trajectories of

$$
\dot{z}(t)=A_{i} z(t)+B_{i} z\left(t-\tau_{i}\right)+\delta_{i}(t)
$$

for all piecewise continuous functions $\delta_{i}:[0,+\infty) \rightarrow \mathbb{R}^{n}$ satisfies $\dot{V}_{i} \leq \alpha_{i} V_{i}\left(z_{t}\right)+\gamma_{i}\left|\delta_{i}(t)\right|^{2}$ for all $t \geq 0$. Moreover, there are constants $\Psi_{1}>0$ and $\Psi_{2}>0$ such that for all $\phi \in C_{\text {in }}$ and all $i \in\{1, \ldots, k\}$, we have

$$
\Psi_{1}|\phi(0)|^{2} \leq V_{i}(\phi) \leq \Psi_{2}|\phi|_{\left[-\tau_{b}, 0\right]}^{2} .
$$

Also, the $\alpha_{i}$ 's satisfy Assumption 3 for the switching signal $\sigma$. Finally, there is a constant $\mu>1$ such that $V_{i}(\phi) \leq$ $\mu V_{j}(\phi)$ for all $(i, j) \in\{1, \ldots, m\}^{2}$ and $\phi \in C_{\text {in }}$.

Assumption 7: Assumption 4 holds, where $\nu$ is a constant that satisfies (8) as before, and $\mu$ and the $\alpha_{i}$ 's are defined as in Assumption 6, and the $\beta_{i}$ 's are defined by

$$
\beta_{i}=\frac{\gamma_{i}\left[\bar{a}+\bar{b}+b_{b} \bar{\tau}\left(a_{b}+b_{b}\right)\right]^{2}}{\Psi_{1}}
$$

for $i=1,2, \ldots, k$.

We also assume that the initial functions for (21) are constant at time 0 . While we can choose

$$
\begin{aligned}
& \bar{a}=a_{b}+\max _{i}\left|A_{i}\right|, \bar{b}=b_{b}+\max _{i}\left|B_{i}\right|, \\
& \text { and } \bar{\tau}=\tau_{b}+\tau^{o} \text { where } \tau^{o}=\max _{i} \tau_{i}
\end{aligned}
$$

in (24), the conditions we placed on $\bar{a}, \bar{b}$, and $\bar{\tau}$ above are more general and so can facilitate checking Assumption 4.

\section{B. Stability Theorem for the LTV System}

Theorem 2: If the system (21) satisfies Assumptions 5-7, then (21) is globally exponentially stable to 0 .

Proof. We can rewrite (21) as

$$
\begin{aligned}
& \dot{x}(t)=A_{j} x(t)+B_{j} x\left(t-\tau_{j}\right)+\left[A(t)-A_{j}\right] x(t) \\
& +\left[B(t)-B_{j}\right] x\left(t-\tau_{j}\right) \\
& +B(t)\left[x(t-\tau(t))-x\left(t-\tau_{j}\right)\right]
\end{aligned}
$$

for each $j \in\{1,2, \ldots, k\}$. Consequently, since we assumed that the initial functions for (21) are constant at time 0 , the system (21) can be represented as the switched system

$$
\dot{x}(t)=A_{\sigma(t)} x(t)+B_{\sigma(t)} x\left(t-\tau_{\sigma(t)}\right)+\Lambda\left(t, \sigma(t), x_{t}\right),
$$

where $\Lambda\left(t, \sigma(t), x_{t}\right)$ consists of the last three terms in (29) with $j=\sigma(t)$. Then

$$
\begin{aligned}
& \left|\Lambda\left(t, j, x_{t}\right)\right| \leq\left|\left[A(t)-A_{j}\right] x(t)\right|+\left|\left[B(t)-B_{j}\right] x\left(t-\tau_{j}\right)\right| \\
& +|B(t)| \int_{t-\max \left\{\tau_{j}, \tau(t)\right\}}^{t-\min \left\{\tau_{j}, \tau(t)\right\}}|[A(\ell) x(\ell)+B(\ell) x(\ell-\tau(\ell))]| \mathrm{d} \ell
\end{aligned}
$$

holds for all $t \in\left[t_{j}, t_{j+1}\right)$ and all $j \in \mathbb{Z}_{\geq 0}$.

Then our choices of the constants give

$$
\begin{aligned}
\left|\Lambda\left(t, \sigma(t), x_{t}\right)\right| \leq & \bar{a}|x(t)|+\bar{b}\left|x\left(t-\tau_{\sigma(t)}\right)\right| \\
& +b_{b} \bar{\tau}\left(a_{b}+b_{b}\right)|x|_{\left[t-2 \tau_{b}-\tau^{o}, t\right]} \\
\leq & {\left[\bar{a}+\bar{b}+b_{b} \bar{\tau}\left(a_{b}+b_{b}\right)\right] } \\
& \times|x|_{\left[t-2 \tau_{b}-\tau^{o}, t\right]}
\end{aligned}
$$

holds for all $t \geq 0$. Hence, Assumption 6 gives

$$
\begin{aligned}
& \dot{V}_{\sigma(t)}(t) \leq \alpha_{\sigma(t)} V_{\sigma(t)}\left(x_{t}\right) \\
& +\frac{\gamma_{\sigma(t)}\left[\bar{a}+\bar{b}+b_{b} \bar{\tau}\left(a_{b}+b_{b}\right)\right]^{2}}{\Psi_{1}} \Psi_{1}|x|_{\left[t-2 \tau_{b}-\tau^{o}, t\right]}^{2}
\end{aligned}
$$

for all $t \geq 0$. Hence, Assumptions 1-4 are satisfied by (30), with $\tau_{b}$ replaced by $2 \tau_{b}+\tau^{0}$ and

$$
W(x)=\Psi_{1}|x|^{2} .
$$

Then Theorem 1 implies that (30) is uniformly globally asymptotically stable. In fact, the proof of Theorem 1 provides an exponential decay estimate on $U\left(t, x_{t}\right)$. Using (26), we deduce that (21) is uniformly globally exponentially stable to 0 . This completes the proof.

Remark 3: Theorem 2 remains true (with the same proof) if we remove the requirement in Assumption 5 that $A, B$, and $\tau$ all have a common period $\mathcal{P}$ (and keep the rest of Assumptions 5-7 the same, with $a_{b}, b_{b}$, and $\tau_{b}$ defined as before) but make the following changes in the notation in Assumptions 6-7: We (i) redefine the $\left(A_{i}, B_{i}, \tau_{i}\right)$ 's in Assumptions 6-7 for $i=1,2, \ldots, k$ to be any triples in $\mathbb{R}^{n \times n} \times \mathbb{R}^{n \times n} \times[0,+\infty)$ for any choice of $k \in \mathbb{N}$, (ii) replace $\sigma$ by any switching signal $\sigma:[0,+\infty) \rightarrow\{1,2, \ldots, k\}$, and then (iii) choose any nonnegative constants $\bar{a}, \bar{b}$, and $\bar{\tau}$ such that $\left|A(t)-A_{\sigma(t)}\right| \leq \bar{a},\left|B(t)-B_{\sigma(t)}\right| \leq \bar{b}$, and $\left|\tau(t)-\tau_{\sigma(t)}\right| \leq \bar{\tau}$ hold for all $t \geq 0$ as before. This makes our work applicable to much more general systems with nonperiodic coefficient matrices.

\section{Example: Periodic System}

We apply our work to the switched linear system

$$
\dot{x}(t)=A_{\sigma(t)} x(t)+B x(t-\tau(t))
$$

with $x$ valued in $\mathbb{R}^{2}$, a piecewise continuous $\tau$ valued in $\left[0, \tau_{b}\right]$ for any bound $\tau_{b}>0$,

$$
A_{1}=-I_{2}, A_{2}=\left[\begin{array}{ll}
-2 & -1 \\
-1 & -2
\end{array}\right] \text {, and } B=\left[\begin{array}{ll}
b & b \\
b & b
\end{array}\right]
$$

with $b \geq 0$ being a constant, and $\sigma:[0,+\infty) \rightarrow\{1,2\}$ being periodic of some period $\mathcal{P}>0$ and defined by $\sigma(t)=1$ when $t \in[0, \mathcal{P} / 2)$ and $\sigma(t)=2$ when $t \in[\mathcal{P} / 2, \mathcal{P})$, and $\tau$ having period $\mathcal{P}$. Assume that

$$
P \geq \frac{2 \tau_{b}}{3} .
$$

Our conditions to follow will ensure that the origin of (34) is uniformly globally asymptotically stable.

Setting $V_{1}(x)=\sqrt{3}|x|^{2} / 2$, it follows that for all $t \in$ $\left[i \mathcal{P}, \frac{\mathcal{P}}{2}+i \mathcal{P}\right)$ and $i \in \mathbb{Z}_{\geq 0}$, we have

$$
\dot{V}_{1}(t)=\frac{\sqrt{3}}{2}\left(-2\left(\left|x_{1}(t)\right|^{2}+\left|x_{2}(t)\right|^{2}\right)\right)+\sqrt{3} b z(t) z(t-\tau(t))
$$

where $z(t)=x_{1}(t)+x_{2}(t)$. Since

$$
\begin{aligned}
& z(t) z(t-\tau(t)) \leq \sup _{\ell \in\left[t-\tau_{b}, t\right]}|z(\ell)|^{2} \\
& \leq 2 \sup _{\ell \in\left[t-\tau_{b}, t\right]}\left(\left|x_{1}(t)\right|^{2}+\left|x_{2}(t)\right|^{2}\right),
\end{aligned}
$$


we have

$$
\dot{V}_{1}(t) \leq-2 V_{1}(x(t))+4 b \sup _{\ell \in\left[t-\tau_{b}, t\right]} V_{1}(x(\ell))
$$

along all solutions of (34). Defining

$$
V_{2}(x)=x_{1}^{2}+x_{1} x_{2}+x_{2}^{2},
$$

it follows that when $t \in\left[i \mathcal{P}+\frac{\mathcal{P}}{2}, i \mathcal{P}+\mathcal{P}\right)$, we have

$$
\begin{aligned}
\dot{V}_{2}(t)= & -5\left(x_{1}^{2}(t)+x_{2}^{2}(t)\right)-8 x_{1}(t) x_{2}(t) \\
& +3 b z(t-\tau(t)) z(t)
\end{aligned}
$$

for all $i \in \mathbb{Z}_{\geq 0}$. Since

$$
2 V_{2}(x) \leq 5|x|^{2}+8 x_{1} x_{2}
$$

holds for all $x \in \mathbb{R}^{2}$, we can now use (37) to obtain

$$
\dot{V}_{2}(t) \leq-2 V_{2}(x(t))+(4 \sqrt{3}) b \sup _{\ell \in\left[t-\tau_{b}, t\right]} V_{1}(x(\ell))
$$

for all $t \in\left[i \mathcal{P}+\frac{\mathcal{P}}{2}, i \mathcal{P}+\mathcal{P}\right)$ and $i \in \mathbb{Z}_{\geq 0}$. Moreover,

$$
V_{2}(x) \leq \sqrt{3} V_{1}(x) \text { and } V_{1}(x) \leq \sqrt{3} V_{2}(x)
$$

for all $x \in \mathbb{R}^{2}$. Using our notation from Assumptions 12, we can then choose $W=V_{1}, \mathcal{T}_{2}=\mathcal{P} / 2$, any constant $\mathcal{T}_{1} \in(0, \mathcal{P} / 2), \mu=\sqrt{3}, \alpha_{1}=-2, \alpha_{2}=-2, \beta_{1}=4 b$, and $\beta_{2}=(4 \sqrt{3}) b$. Also, choosing $T=2 \mathcal{P}$, our assumption that $\mathcal{P} \geq 2 \tau_{b} / 3$ gives

$$
T=\mathcal{P} / 2+3 \mathcal{P} / 2 \geq \mathcal{P} / 2+\tau_{b}=\mathcal{T}_{2}+\tau_{b},
$$

and

$$
\int_{t-T}^{t} a(\ell) \mathrm{d} \ell=-2 T=-4 \mathcal{P}
$$

for all $t \geq T$, so Assumption 3 holds with $\lambda(T)=4 \mathcal{P}$. Moreover, we can pick $\nu=(2 \sqrt{3}) b\left(1-e^{-4 \mathcal{P}}\right)$, and $L(2 \mathcal{P})=$ 4.

From (9) and Theorem 1, it follows that if

$$
\begin{aligned}
& \mu^{L(T)+1} e^{-\lambda(T)}+\left[\frac{\mu^{L(T)+2}-1}{\mu-1}-L(T)-1\right] \nu \mu \\
& =9 \sqrt{3} e^{-4 \mathcal{P}}+\left[\frac{26}{\sqrt{3}-1}-5\right] 6 b\left(1-e^{-4 \mathcal{P}}\right)<1
\end{aligned}
$$

then (34) is uniformly globally asymptotically stable to 0 . Hence, this system is uniformly globally asymptotically stable to the origin if $\mathcal{P}$ is large enough and $b$ is sufficiently small. The largest $b$ obtained from (46) as a function of $\mathcal{P}$ is shown in Figure 1 as the function $b_{\max }$.

Note that this result may appear to be independent of $\tau_{b}$, but it is not, since our assumption $\mathcal{P} \geq 2 \tau_{b} / 3$ from (36) couples the condition (46) with $\tau_{b}$. When $b=0$, i.e., in the no delay case, the switched system is uniformly globally asymptotically stable to the origin, because $\dot{x}=A_{1} x$ and $\dot{x}=A_{2} x$ have a common Lyapunov function $V(x)=|x|^{2}$.

\section{CONCLUSION}

Our new trajectory based approach allowed us to prove globally asymptotic stability of a large class of switched time-varying systems with time-varying delays. Our results apply to a wide family of systems for which no other

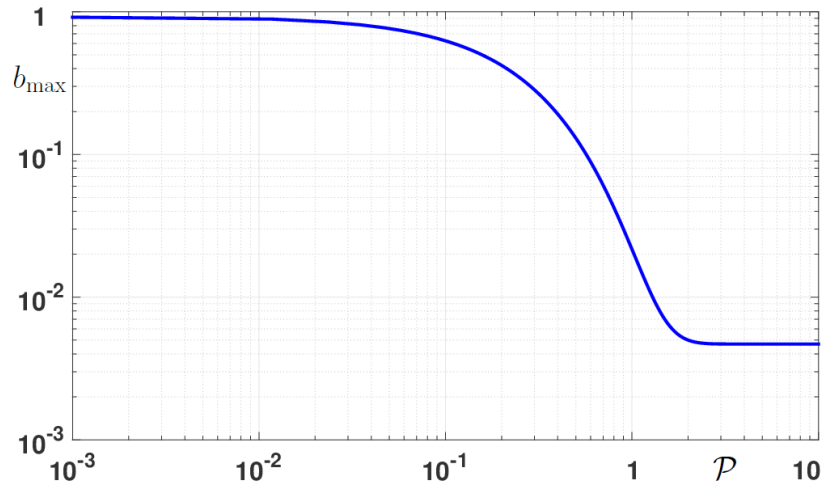

Fig. 1. $b_{\max }$ versus $\mathcal{P}$.

stability analysis technique was available. When applied to linear systems, our method only requires constructing simple Lyapunov-Krasovskii functionals for time invariant systems. In future work, we hope to use the results of this paper to obtain control designs under robustness or optimality conditions, including input-to-state stability results.

\section{REFERENCES}

[1] S. Caliskan, H. Özbay, and S-I. Niculescu. Dwell-time computation for stability of switched systems with time delays. IET Control Theory and Applications, 7(10):1422-1428, 2013.

[2] D. Cheng, J. Wang, and X. Hu. An extension of LaSalle's Invariance Principle and its application to multi-agent consensus. IEEE Transactions on Automatic Control, 53(7):1765-1770, 2008.

[3] A. Halanay. Differential Equations, Stability, Oscillations, Time Lags. Academic Press, New York, 1966.

[4] Z-P. Jiang, Y. Lin, and Y. Wang. Stability analysis for switched systems with ISS and unstable time-delayed subsystems. In Proceedings of the 28th Chinese Control and Decision Conference, Yinchuan, China, 2016, pp. 905-909.

[5] A. Koru, A. Delibasi, and H. Özbay. Dwell time-based stabilisation of switched delay systems using free-weighting matrices. International Journal of Control, DOI: 10.1080/00207179.2016.1266515, 2017.

[6] D. Liberzon. Switching in Systems and Control. Birkhauser, Berlin, Germany, 2003.

[7] J. Mancilla-Aguilar and R. Garcia. On converse Lyapunov theorems for ISS and iISS switched nonlinear systems. Systems and Control Letters, 42(1):47-53, 2001.

[8] F. Mazenc and M. Malisoff. Trajectory based approach for the stability analysis of nonlinear systems with time delays. IEEE Transactions on Automatic Control, 60(6):1716-1721, 2015.

[9] P. Pepe. Stabilization in the sample-and-hold sense of nonlinear retarded systems. SIAM Journal on Control and Optimization, 52(5):30533077, 2014.

[10] Z. Sun and S. Ge. Stability Theory of Switched Dynamical Systems. Communications and Control Engineering Series. Springer, New York, 2011.

[11] L. Vu and K. Morgansen. Stability of time-delay feedback switched linear systems. IEEE Transactions on Automatic Control, 55(10):23852390, 2010

[12] Y-E. Wang, X-L. Sun, and F. Mazenc. Stability of switched nonlinear systems with delay and disturbance. Automatica, 69:78-86, 2016.

[13] G. Xie and L. Wang. Periodical stabilization of switched linear systems. Journal of Computational and Applied Mathematics, 181:176187, 2005.

[14] D. Xie and Y. Wu. Stabilisation of time-delay switched systems with constrained switching signals and its applications in networked control systems. IET Control Theory and Applications, 4(10):2120$2128,2010$.

[15] P. Yan and H. Özbay. Stability analysis of switched time-delay systems. SIAM Journal on Control and Optimization, 47(2):936-949, 2008

[16] B. Zhou and A. Egorov. Razumikhin and Krasovskii stability theorems for time-varying time-delay systems. Automatica, 71:281-291, 2016. 